\title{
Impact of anesthesia on pathophysiology and mortality following subarachnoid hemorrhage in rats
}

\author{
Konstantin Hockel ${ }^{1}$, Raimund Trabold ${ }^{1,2}$, Karsten Schöller ${ }^{1,2}$, Elisabeth Török ${ }^{1}$ and Nikolaus Plesnila ${ }^{1,2,3^{*}}$
}

\begin{abstract}
Background: Anesthesia is indispensable for in vivo research but has the intrinsic potential to alter study results. The aim of the current study was to investigate the impact of three common anesthesia protocols on physiological parameters and outcome following the most common experimental model for subarachnoid hemorrhage (SAH), endovascular perforation.

Methods: Sprague-Dawley rats $(\mathrm{n}=38)$ were randomly assigned to (1) chloral hydrate, (2) isoflurane or (3) midazolam/medetomidine/fentanyl (MMF) anesthesia. Arterial blood gases, intracranial pressure (ICP), mean arterial blood pressure (MAP), cerebral perfusion pressure (CPP), and regional cerebral blood flow (rCBF) were monitored before and for 3 hours after SAH. Brain water content, mortality and rate of secondary bleeding were also evaluated.
\end{abstract}

Results: Under baseline conditions isoflurane anesthesia resulted in deterioration of respiratory parameters (arterial $\mathrm{pCO}_{2}$ and $\mathrm{pO}_{2}$ ) and increased brain water content. After $\mathrm{SAH}$, isoflurane and chloral hydrate were associated with reduced MAP, incomplete recovery of post-hemorrhagic rCBF (23 $\pm 13 \%$ and $87 \pm 18 \%$ of baseline, respectively) and a high anesthesia-related mortality (17 and 50\%, respectively). Anesthesia with MMF provided stable hemodynamics (MAP between 100-110 mmHg), high post-hemorrhagic rCBF values, and a high rate of rebleedings (> 50\%), a phenomenon often observed after SAH in humans.

Conclusion: Based on these findings we recommend anesthesia with MMF for the endovascular perforation model of SAH.

Keywords: Subarachnoid hemorrhage, Rat, Experimental, Anesthesia, Isoflurane, Chloral hydrate, Medetomidine, Brain edema, Cerebral blood flow, Re-bleeding

\section{Background}

Experimental animal models are widely used to study the pathophysiology of subarachnoid hemorrhage (SAH) [1-4]. The induction of experimental SAH is performed by invasive surgical techniques which require continuous multimodal monitoring and adequate sedation and analgesia. Ventilation and, hence, blood gases can be controlled by intubation and mechanical ventilation [5], however, other parameters which are known to critically determine the outcome following experimental SAH

\footnotetext{
* Correspondence: nikolaus.plesnila@med.uni-muenchen.de 'Laboratory of Experimental Neurosurgery, Institute for Surgical Research, Munich, Germany

Full list of author information is available at the end of the article
}

such as intracranial pressure (ICP), mean arterial blood pressure (MAP) and regional cerebral blood flow (rCBF) $[1,2,6-9]$ may well be influenced by the applied anesthetic protocol as already demonstrated for experimental models of cerebral ischemia [5] and traumatic brain injury $[10,11]$.

Various inhaled or injectable anesthetics have been used in experimental $\mathrm{SAH}$, e.g. halothane, isoflurane [alone or in combination with nitrous oxide $\left.\left(\mathrm{N}_{2} \mathrm{O}\right)\right][1,3,4,12,13]$, chloral hydrate, barbiturates [14-16], ketamine/xylazine or midazolam/medetomedine/fentanyl (MMF) [17-19]. Although experimental SAH models are known to suffer from some variability $[2,3,9]$, little is know to which degree the used anesthetic protocol contributes to this phenomenon.

\section{Biomed Central}


Therefore, we investigated the effect of three well established and frequently used standard anesthesia protocols, namely chloral hydrate, isoflurane, and MMF on animal physiology, mortality and brain water content before and after experimental SAH in rats. The ultimate aim of the study was to determine how anesthesia influences the outcome of experimental SAH and, if significant differences are found between different anesthesia protocols, which protocol has the least influence on the pathophysiology induced by the endovascular perforation model, the most frequently used and the presumably most clinically relevant model of subarachnoid hemorrhage.

\section{Methods}

In this study we used 38 male Sprague-Dawley rats (250300 g body weight), purchased from Charles River Laboratories, Sulzfeld, Germany. Eight animals died during experiments (see below), i.e. 30 animals were included in the final analysis. Animals had free access to pellet food until 12 hours prior to surgery. Water was accessible at all time. All experiments were approved by the Ethics Committee of the District Government of Upper Bavaria, Germany.

\section{Anesthesia}

Anesthesia protocols were chosen based on the frequency of their use with the endovascular perforation model of $\mathrm{SAH}$ (e.g. isoflurane), on their availability (e.g. halothane was not included in the analysis since its production for human use was discontinued resulting in a reduced availability for many laboratories), on their known small influence of cerebral blood flow (chloral hydrate and midazolam/medetomidine/fentanyl), on the ease of their termination (isoflurane and midazolam/medetomidine/ fentanyl), and on their level of standardization in experimental and veterinary medicine (all three protocols).

Dose regiments were based on the scientific and veterinary literature and our own experience during the past 5 (midazolam/medetomidine/fentanyl) to 20 (chloral hydrate and isoflurane) years. A similar level of sedation between the three protocols was achieved by using the lowest dose necessary to reach surgical anesthesia (as verified by the tail pinch test and concomitant observation of withdrawal reflexes and systemic blood pressure).

Anesthesia was induced by placing animals in a chamber with $4 \%$ isoflurane for 2-3 minutes. Thereafter rats were randomized to one of the three standard anesthesia protocols: (1) Chloral hydrate $(1 \mathrm{ml} / 100 \mathrm{~g}$ body weight of a $3.6 \%$ solution) was injected intraperitoneally (i.p.). Animals were intubated and ventilated with $70 \%$ air and 30\% $\mathrm{O}_{2}$ under control of ventilation pressure and rate (Small animal ventilator KTR-4, Hugo Sachs Elektronik, Germany). A third of the initial dosage was applied every 60 minutes in order to maintain anesthesia. (2) Animals were ventilated with $2 \%$ isoflurane in $70 \% \mathrm{~N}_{2} \mathrm{O}$ and $30 \%$ $\mathrm{O}_{2}$. (3) Midazolam $(2 \mathrm{mg} / \mathrm{kg})$, medetomidine $(0.15 \mathrm{mg} /$ $\mathrm{kg})$ and fentanyl $(0.0075 \mathrm{mg} / \mathrm{kg})$ were given by intraperitoneal injection. Animals were ventilated with $70 \%$ air and $30 \% \mathrm{O}_{2}$. A third of the initial dosage was applied for maintenance of anesthesia every 40 minutes. Atropine (150 mg/kg bodyweight) was injected subcutaneously in all experimental groups to inhibit salivary secretion and induce bronchodilation.

\section{Monitoring}

The tail artery was canulated for continuous measurement of mean arterial blood pressure (MAP) and for blood sampling throughout the experiment. Arterial blood gases, $\mathrm{pH}$, serum glucose and lactate were analyzed every 30 minutes. Ventilation rate (in ventilations per minute $=\mathrm{vpm}$ ) and pressure were adapted in order to maintain physiological arterial $\mathrm{pCO}_{2}$ values (35-45 $\mathrm{mmHg}$ ). Temporal muscle and rectal probes were used to monitor brain and body temperature, respectively. A thermostatically regulated, feedback-controlled heating pad and lamp were used to maintain rectal and brain temperature at $37^{\circ} \mathrm{C}$.

Intracranial pressure (ICP) was continuously measured using a Codman ICP microsensor (Johnson \& Johnson Medical Limited, Berkshire, UK). After drilling a burr hole over the right parietal cortex under constant cooling, the probe was advanced $2 \mathrm{~mm}$ into the brain and fixed with dental cement. Cerebral perfusion pressure (CPP) was calculated using the following formula: $\mathrm{CPP}=$ MAP - ICP.

A 2-channel laser-Doppler flowmeter (LDF; MBF3D, Moor Instruments Ltd.) was used for continuous monitoring of regional cerebral blood flow ( $\mathrm{rCBF}$ ) in the territory of the middle cerebral artery (MCA) of both hemispheres as previously described $[4,13]$.

\section{Induction of SAH}

SAH was induced by endovascular puncture, one of the most widely used models for experimental SAH [1-3], as previously described [4]. Briefly, a 3-0 monofilament was advanced via the external carotid artery (ECA) into the internal carotid artery (ICA) until increase of ICP and bilateral decrease of $\mathrm{rCBF}$ indicated SAH. Subsequently, the suture was withdrawn and the ECA ligated.

\section{Experimental groups}

In a first series of experiments blood gases, MAP, ICP, $\mathrm{CPP}$ and rCBF were continuously monitored for 3 hours under physiological conditions in all three experimental groups ( $\mathrm{n}=5$ each). Afterwards animals were sacrificed for quantification of brain water content. In a second series animals were randomly assigned to one of the three anesthesia groups $(\mathrm{n}=5 \mathrm{each})$ and $\mathrm{SAH}$ was induced. All 
physiologic parameters were recorded under baseline conditions and continuously over 3 hours after SAH. At the end of observation time anesthesia was terminated and animals were tested for signs of awakening, i.e. adequate spontaneous respiration. Animals that died during surgery or exhibited excessive re-bleedings were replaced until the final group size was $n=5$ in all groups. Subsequently, animals were re-anesthetized and sacrificed for quantification of brain water content.

\section{Quantification of brain water content}

Brains were removed and the hemispheres were separated and weighed to assess their wet weight (WW). Thereafter, the hemispheres were dried for $24 \mathrm{~h}$ at $110^{\circ} \mathrm{C}$ and their dry weight (DW) was determined. Hemispheric water content (\%) was calculated using the following formula: $((W W-D W) / W W) \times 100$.

\section{Statistical analysis}

Statistical analysis was performed with SigmaStat 3.1 (SPSS Science Inc., Chicago, IL, USA). Blood gas parameters were analyzed with Kruskal-Wallis - ANOVA on ranks followed by Student-Newman-Keuls as post hoc test, and for physiologic parameters ANOVA on ranks/ Dunn's Method was applied. Two points in time of the same group were compared with Friedman - repeated measures ANOVA on ranks. Statistical significance of results was assumed at $\mathrm{p}<0.05$ and a power of $>0.8$. Data are presented as means \pm SEM if not otherwise indicated.

\section{Results}

All three anesthesia protocols provided sufficient sedation and analgesia for surgical intervention - the animals showed no signs of untimely awakening or discomfort at any time. Pinching of the hind paw did not trigger a withdrawal reflex or an increase in blood pressure.

\section{Ventilation rate and blood gases}

Under physiological conditions anesthesia with isoflurane required a significant increase of ventilation rate during the 180 minutes of observation to keep $\mathrm{PaCO}_{2}$ within normal limits $(58 \pm 2 \mathrm{vpm}$ vs. $27 \pm 1 \mathrm{vpm}$ in rat anesthetized with MMF; $p<0.05$; Table 1).

After SAH induction, anesthesia with isoflurane and chloral hydrate required an increase of the ventilation rate that reached statistical significance $(p<0.05)$ compared to MMF after 180 minutes $(47 \pm 3,42 \pm 4$, and $34 \pm 3 \mathrm{vpm}$, respectively) (Table 1 ). Ventilation pressure was not different between groups $(12.6 \pm 0.2,12.7 \pm 0.2$ and $12.6 \pm 0.4 \mathrm{cmH}_{2} \mathrm{O}$ for chloral hydrate, isoflurane and $\mathrm{MMF}$, respectively).

Arterial $\mathrm{pCO}_{2}$ and $\mathrm{pH}$ were not significantly different between the three anesthesia protocols under physiological conditions or after $\mathrm{SAH}$ induction. Arterial $\mathrm{pO}_{2}$ in the isoflurane group, however, decreased significantly during the course of the experiment $(p<0.05)$ and was significantly lower compared to chloral hydrate and MMF $(p<0.05)$ after 60 and 180 minutes under physiological conditions $(116.0 \pm 6.3,89.6 \pm 2.8$, and $135.6 \pm 8.8 \mathrm{mmHg}$ for chloral hydrate, isoflurane and MMF, respectively). Accordingly, in $\mathrm{SAH}$ groups a decline in arterial $\mathrm{pO}_{2}$ was observed for isoflurane $(p<0.05)$, with values significantly different from the chloral hydrate and MMF group at 60 minutes after SAH (Table 1).

\section{ICP, MAP, CPP, and rCBF}

There was no significant difference in ICP (values around $5 \mathrm{mmHg}$ ) between the three groups under physiological conditions. SAH resulted in an immediate increase in ICP to a peak of $42 \pm 8,28 \pm 4$ and $61 \pm$ $17 \mathrm{mmHg}$ in the chloral hydrate, isoflurane and MMF group, respectively (Figure 1A). This peak was followed by a plateau of $15-20 \mathrm{mmHg}$ that was reached after 15 minutes in all groups.

Under physiological conditions animals subjected to MMF anesthesia exhibited a significantly $(p<0.05)$ higher MAP ( 100 mmHg) compared to the groups anesthetized with chloral hydrate $(\sim 80 \mathrm{mmHg})$ or isoflurane $(\sim 70$ $\mathrm{mmHg})$. After SAH MAP remained significantly $(p<0.05)$ elevated in the MMF group (Figure $1 \mathrm{~B}$ ). Of note, the sudden rise in ICP after SAH triggered a Cushing reflex in MMF anesthetized animals: MAP acutely increased by $\sim 20 \mathrm{mmHg}$ (from $98 \pm 7$ to $118 \pm 12 \mathrm{mmHg}$ ) and returned to baseline values 15 minutes later. In animals anesthetized with chloral hydrate or isoflurane, however, this expected physiological increase in blood pressure was not observed. On the contrary, MAP actually dropped by $20 \mathrm{mmHg}$ and recovered only slowly thereafter (Figure 1B) indicating that chloral hydrate and isoflurane interfere with physiological blood pressure regulation.

Due to the higher MAP under physiological conditions, CPP was significantly $(p<0.05)$ higher in MMF anesthetized rats $(\sim 95 \mathrm{mmHg})$ compared to those animals which received chloral hydrate $(\sim 75 \mathrm{mmHg})$ or isoflurane $(\sim 65 \mathrm{mmHg})$. Following $\mathrm{SAH}, \mathrm{CPP}$ decreased by $\sim 30-45 \mathrm{mmHg}$ in all experimental groups. In the MMF group, CPP returned to near baseline values of 88 $\pm 7 \mathrm{mmHg}$ within 5 minutes after SAH. For chloral hydrate and isoflurane a constantly reduced CPP was observed after SAH (51 \pm 7 and $41 \pm 3 \mathrm{mmHg}$, respectively; $p<0.05$ vs. MMF).

Anesthesia with chloral hydrate or MMF did not increase $\mathrm{rCBF}$ during the 3 hour observation time $(7 \pm$ $13 \%$ and $5 \pm 6 \%$ rCBF increase vs. 30 min after initiation of anesthesia, respectively), while in isoflurane anesthetized rats $\mathrm{rCBF}$ increased by over $1 / 3$ (35 $\pm 22 \% \mathrm{rCBF}$ increase vs. $30 \mathrm{~min}$ after initiation of anesthesia). 
Table 1 Physiological parameters during surgical preparation, before, and after SAH

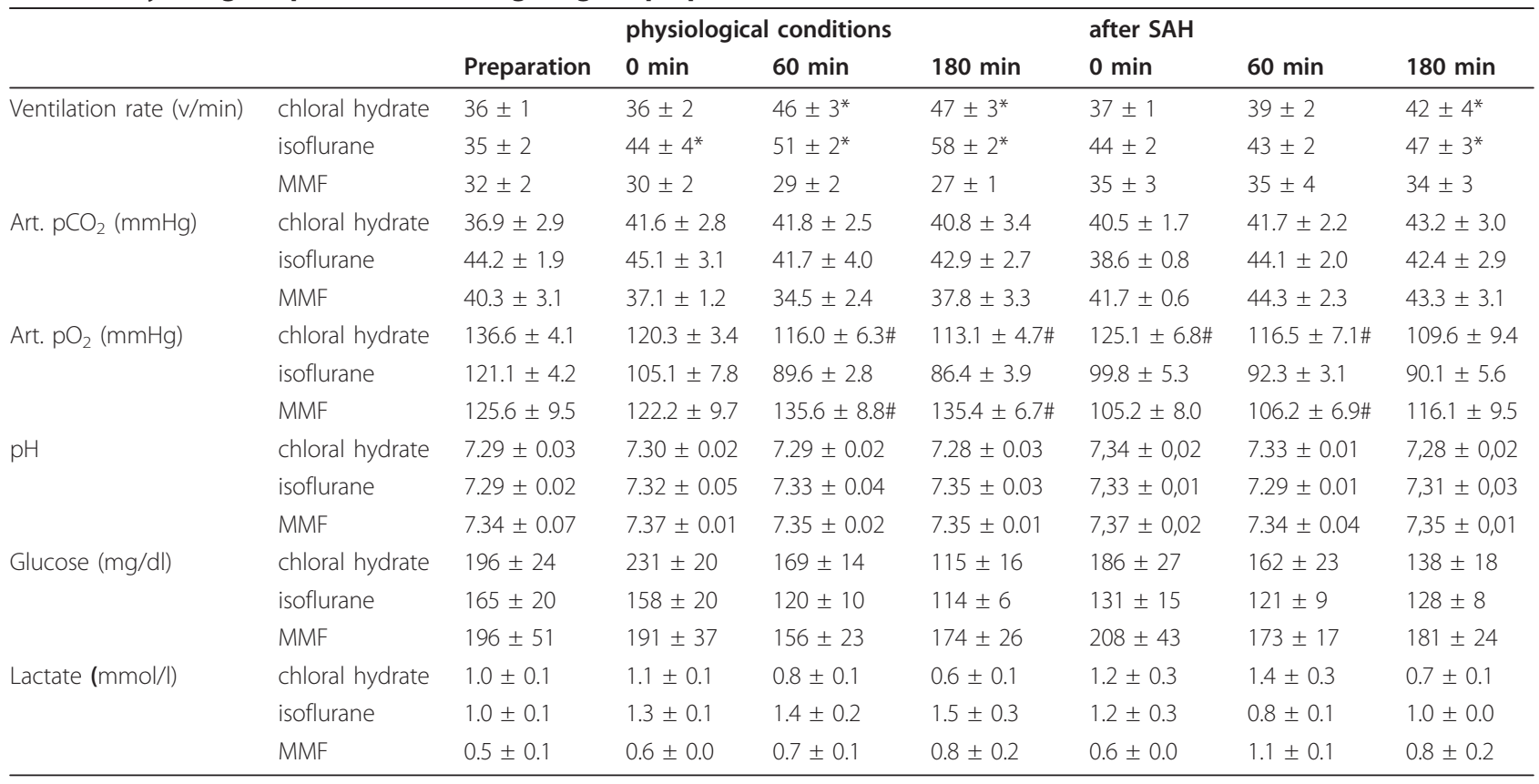

In all animals $\mathrm{SAH}$ resulted in a sharp decline of ipsilateral $\mathrm{rCBF}(p<0.05)$ to $\sim 10-30 \%$ of baseline (Figure 2 ). No recovery was observed in the isoflurane group while in MMF and chloral hydrate anesthetized rats $\mathrm{rCBF}$ recovered to almost baseline values (92 $\pm 13 \%$ and $87 \pm$ $18 \%$, respectively). Contralateral $\mathrm{rCBF}$ recovered within the 3 hours observation period in all groups.

\section{Brain water content (BWC)}

Following 4.5 hours of anesthesia, brain water content was in the physiological range in the MMF group $(78.8 \pm 0.1 \%)$ but increased in animals anesthetized with chloral hydrate or isoflurane $(p<0.05 ; 79.5 \pm$ $0.1 \%$ and $79.6 \pm 0.1 \%$, respectively; Figure 3 ) suggesting either opening of the blood brain barrier or, more likely, increasing cerebral blood volume due to anesthesia-induced vasodilation.

Three hours after induction of SAH brain water content was $79.5 \pm 0.2 \%, 80.1 \pm 0.4 \%$, and $78.9 \pm 0.1 \%$ in the chloral hydrate, isoflurane and MMF groups, respectively. SAH did not result in an increase in brain water content in chloral hydrate and MMF anesthetized animals $\left(0 \%\right.$ and $\left.0.1 \% \triangle B W C^{\text {pre/post }}\right)$, while slight brain edema formation $\left(0.5 \% \Delta \mathrm{BW} \mathrm{C}^{\text {pre/post }}\right)$ was observed in the isoflurane group ( $p<0.05$ vs. MMF; Figure 3 ).

\section{Mortality and re-bleeding after SAH}

There was no mortality during surgical preparation and sham operated animals did not show any mortality.

After SAH, animals anesthetized with chloral hydrate had the highest rate of intraoperative mortality (50\%) followed by animals anesthetized with MMF (29\%) and isoflurane (17\%, Figure 4A).

Significant re-bleedings, which were identified by a recurrent ICP increase of $>20 \mathrm{mmHg}$, were detected in chloral hydrate $(1 / 10,10 \%)$ and $\operatorname{MMF}(4 / 7,57 \%)$ anesthetized animals, in the latter contributing to the mortality in this group (Figure 4B). In total 8 out of the 38 animals used for the current study died prematurely resulting in $\mathrm{n}=30$ animals being included in the final analysis.

\section{Discussion}

In the present study we evaluated the effect of three widely used and well standardized anesthesia protocols on pathophysiology and outcome following subarachnoid hemorrhage $(\mathrm{SAH})$ in rats. Surprisingly, different anesthesia protocols resulted in completely different pathophysiologies following SAH. Isoflurane and chloral hydrate anesthesia resulted in low blood pressure, lack of the expected physiological post-ictal Cushing response, impaired recovery of post-hemorrhagic ischemia and no re-bleedings, while a combination of midazolam, medetomidine and fentanyl (MMF) maintained physiological blood pressure, allowed the rising ICP to trigger the expected increase in systemic blood pressure (Cushing reflex), resulted in complete recovery of post-hemorrhagic cerebral blood flow and caused a significant number of rebleedings, an important characteristic of SAH in humans.

\section{Ventilation and arterial blood gases}

Most agents used for small animal anesthesia cause a dose dependent depression of central respiratory activity 


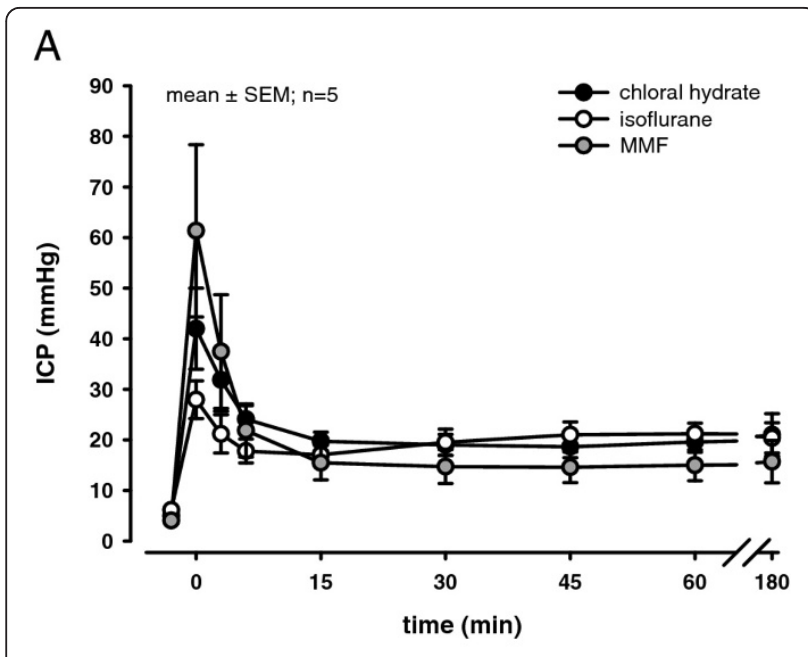

B

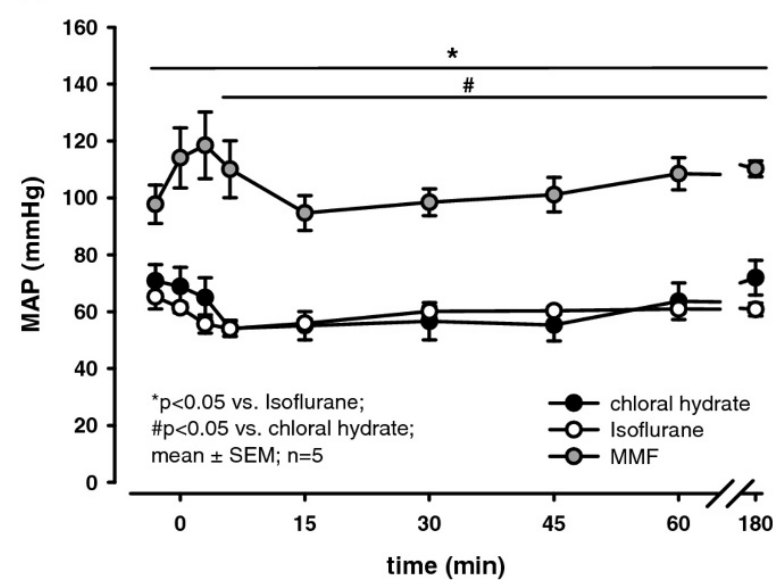

Figure 1 (A) Time course of ICP until 180 minutes after SAH No significant differences were observed between groups. (B) Time course of dynamic MAP changes until 180 minutes after SAH. MAP was significantly higher before and after SAH when animals were anesthetized with MMF as compared to chloral hydrate or isoflurane. SAH induced a Cushing response (transient increase of MAP due to increased ICP) in animals anesthetized with MMF while no such response was observed in the isoflurane or chloral hydrate groups. ICP and MAP were recorded continuously, averaged over 3 minutes, and displayed as means \pm SEM $\left(n=5\right.$ in each group; ${ }^{*} p<$ 0.05 vs. isoflurane, \#p < 0.05 vs. chloral hydrate; Kruskal-Wallis ANOVA on ranks followed by Dunn's post-hoc test).

leading to deterioration in arterial blood gases [20,21]. Therefore, particularly with longer duration experiments, intubation and mechanical ventilation is used to keep blood gases within physiological limits [5]. Animals in the isoflurane group experienced a gradual decrease in arterial $\mathrm{pO}_{2}$ (blood oxygenation) and, similarly to chloral hydrate, an increase in arterial $\mathrm{pCO}_{2}$ that required continuous adjustments of ventilation parameters. Airway irritation and impaired mucocilliary clearance have previously been described for inhalative

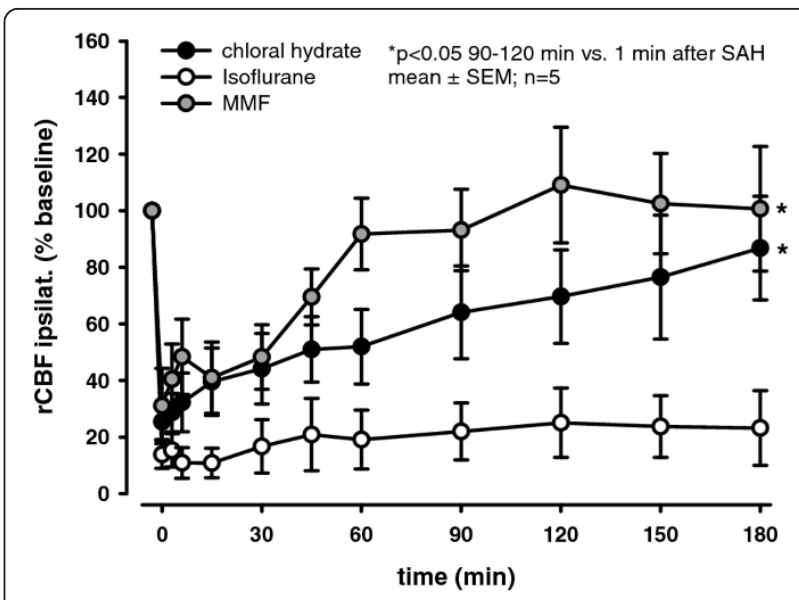

Figure 2 Regional cerebral blood flow of the hemisphere ipsilateral to hemorrhage measured by laser Doppler fluxmetry until 180 minutes after SAH. SAH resulted in an immediate reduction of $\mathrm{rCBF}$ in all groups with a significantly better recovery in the MMF group $\left({ }^{*} p<0.05\right.$ vs. isoflurane). rCBF was recorded continuously, averaged over 3 minutes, and displayed as percentage (\%) of pre-hemorrhagic baseline (means $\pm \mathrm{SEM} ; \mathrm{n}=5$ in each group; Kruskal-Wallis ANOVA on ranks followed by Dunn's post-hoc test).

agents like isoflurane and halothane $[22,23]$ and most probably account for the reduced gas exchange and deterioration in blood gases. Keeping arterial $\mathrm{paCO}_{2}$ within physiological limits is particularly critical following pathological conditions, as carbon dioxide is not

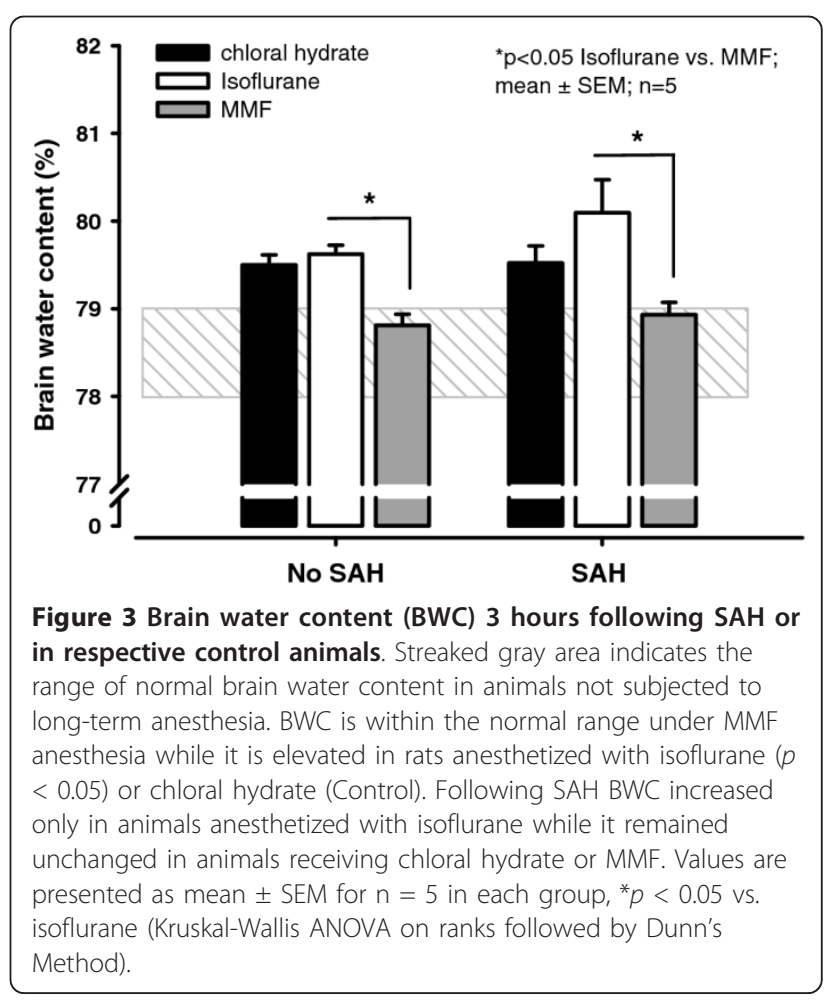




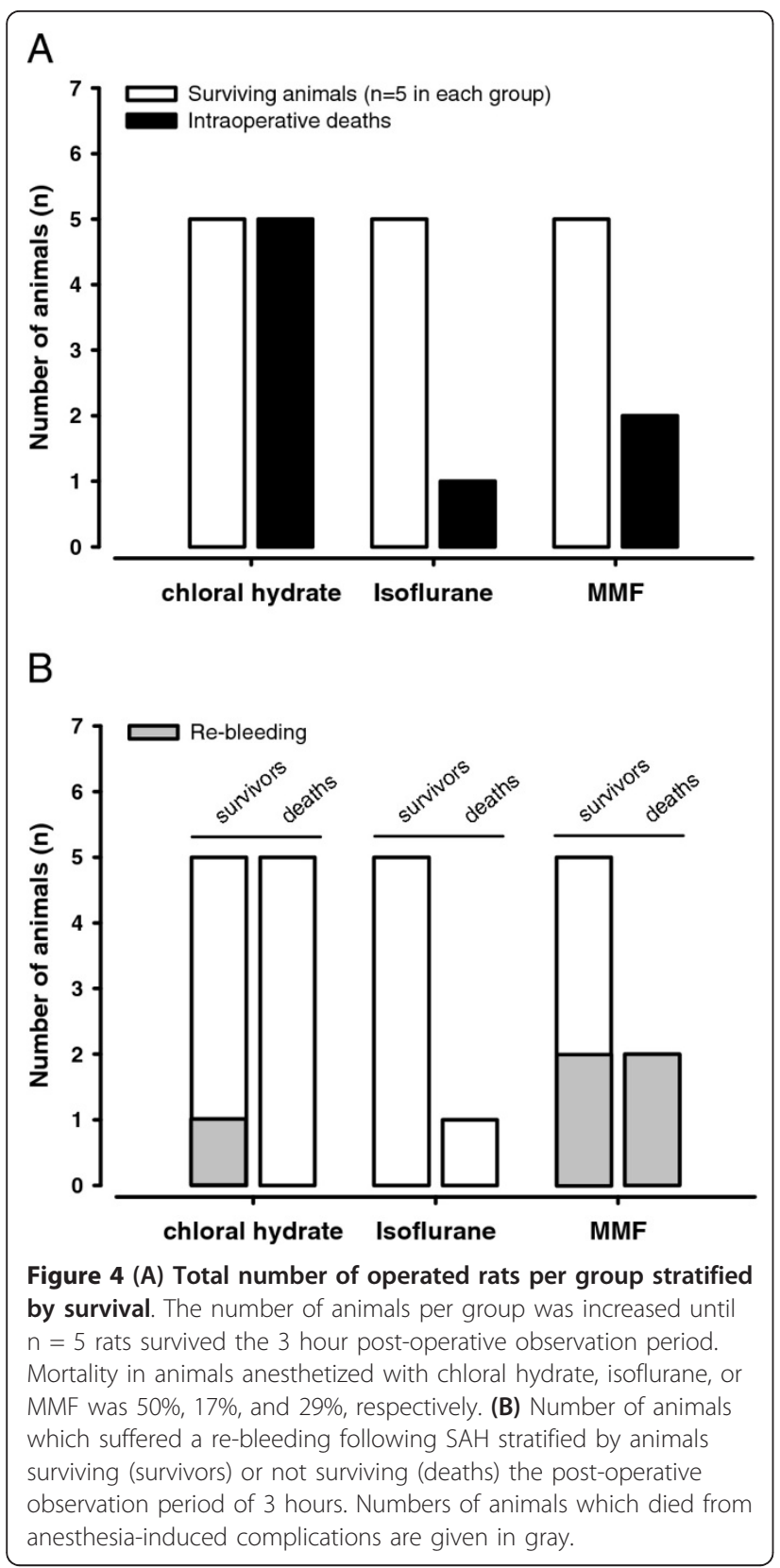

only a potent regulator of cerebrovascular tone [24] but hypercapnia and subsequent acidosis are known to increase ischemic brain injury $[25,26]$. Also, hypoxemia, a symptom of respiratory distress which is frequently observed after SAH $[27,28]$, has been shown to increase the likelihood of a poor outcome [29-31]. In contrast to isoflurane and chloral hydrate the MMF regimen did not affect ventilation; $\mathrm{pO}_{2}$ and $\mathrm{pCO}_{2}$ remained in the physiological range without necessitating constant adjustment of ventilator settings. Therefore, in our hands, MMF anesthesia provided the most stable physiological conditions (especially in combination with mechanical ventilation), allowing prolonged, hassle-free surgery.

\section{MAP, ICP, CPP, and rCBF}

Anesthesia with chloral hydrate or isoflurane caused a reduction of MAP, most likely due to their well known negative effect on cardiac function and vascular tone $[20,32]$. In contrast, MAP remained closest to physiological values of conscious rats $(100-110 \mathrm{mmHg})$ [33] in the MMF group. The difference in MAP amongst the groups correlated with the ICP increase during SAH induction, which implies that the amount and severity of hemorrhage in this model is directly influenced by pre-hemorrhagic MAP. MAP monitoring is, therefore, an absolute prerequisite for the proper and reproducible induction of experimental SAH.

The acute stage of SAH is frequently accompanied by derangements in arterial blood pressure [3,31,34]. A hypertensive reaction, as exhibited in MMF animals, is described as central activation of vasomotor centers to restore cerebral perfusion in the presence of raised ICP, i.e. the well known Cushing reflex [35,36]. The lack of such a robust physiological reflex in the chloral hydrate and isoflurane group suggests that these agents severely affect the cardiovascular system, thereby altering posthemorrhagic pathophysiology in an uncontrolled manner. Our data, therefore, indicate that studies on the pathophysiology of SAH under chloral hydrate or isoflurane anesthesia may need to be re-interpreted carefully, since there is a high probability that they may have been performed under a disturbed cardiovascular baseline.

Even more divergent effects of the three anesthesia protocols were observed when post-hemorrhagic $\mathrm{rCBF}$ was measured. Whilst rats anesthetized with MMF showed an almost complete recovery from SAH-induced ischemia due to high MAP and CCP, rCBF did not recover at all in animals receiving isoflurane anesthesia. Since isoflurane and chloral hydrate have a very similar suppressive effect on systemic blood pressure these findings suggest that isoflurane may have an additional vasodilatatory effect on cerebral vessels, as also suggested by others [37]. The mechanisms responsible for this effect seem to be a selective upregulation of nitric oxide synthesis in the brain by volatile anesthetics [38]. These findings suggest that the use of isoflurane and other volatile anesthetics should be discouraged when studying $\mathrm{SAH}$.

\section{Brain water content}

Our findings demonstrating increased brain water content in rats anesthetized with isoflurane are supported by previous studies where volatile anesthetics increased 
brain water content in healthy rats and dogs [11,39]. These findings are in agreement with the local vasodilatatory effects of isoflurane on the cerebral vasculature; vasodilation increases cerebral blood volume and hence brain water content [40-42].

Isoflurane anesthesia increases brain water content following brain injury, e.g. after experimental traumatic brain injury [11] and after focal cryogenic lesion [43] as also observed in the current study. This is most likely caused by the effect of isoflurane on cerebral blood volume (see above), however, brain damage due to prolonged post-hemorrhagic ischemia/hypoperfusion [44] would also be expected to be involved in the development of brain edema in animals anesthetized with isoflurane.

\section{Mortality}

The two major determinants of post-hemorrhagic mortality in our study were lack of recovery of cerebral hypoperfusion and re-bleeding. Interestingly, the occurrence of hypoperfusion and re-bleeding depended exclusively on the anesthetic protocol used. Hypoperfusion-/ischemicinduced death only occurred after isoflurane and chloral hydrate anesthesia. While isoflurane seems to have a direct CBF-reducing effect (see above), chloral hydrate-induced death was, in most cases, due to episodes of arterial hypotension followed by cessation of rCBF following intraperitoneal re-application of chloral hydrate, as also reported in experimental stroke [45]. This specific problem may, however, be overcome by continuous application of chloral hydrate using a intraperitoneal catheter [46].

Mortality occurring in animals anesthetized with MMF was, in all cases, related to post-hemorrhagic re-bleedings, which are an integral part of the pathophysiology of SAH in humans. The underlying mechanisms of MMFinduced re-bleedings are the maintained MAP and CPP. High MAP and CPP, although beneficial for cerebral perfusion, increase the risk for re-hemorrhages [47] with a high mortality rate [48], as also suggested by our findings. Hence, using MMF for anesthesia during experimental $\mathrm{SAH}$ in rats reproduces an important component of SAH in patient, i.e. delayed re-bleedings, which is usually missing when using when chloral hydrate or isoflurane. Accordingly, only when rats were anesthetized with MMF the pathophysiology of SAH was properly reproduced by the endovascular perforation model.

\section{Handling for general anesthesia in rats}

All three protocols proved to be suitable for surgical anesthesia, however, only MMF appears to not have any negative effect on the pulmonary system, thereby resulting in a far more stable maintenance of arterial $\mathrm{pCO}_{2}$ and $\mathrm{pO}_{2}$ with fewer interventions by the experimentalist compared to isoflurane and chloral hydrate. When considering that MMF anesthesia can be terminated by injection of respective antagonists and that anesthesia under chloral hydrate is difficult to judge and to terminate [49], it seems fair to conclude that MMF is superior to the other two investigated protocols.

\section{Conclusion}

We investigated if and how three widely used and well standardized anesthesia protocols [isoflurane, chloral hydrate, and a combination of midazolam, medetomidine, and fentanyl (MMF)] affect pathophysiology and outcome following experimental SAH in rats. Our experiments demonstrate that when rats are anesthetized with MMF key properties (re-bleedings, Cushing reflex, moderate mortality) known to be integral parts of the pathophysiology of SAH in humans $[47,48,50]$ are replicated by the endovascular perforation model of experimental SAH. Our findings demonstrate that the choice of anesthesia can have a significant impact on animal models of disease and suggest that MMF should be used for future studies using the endovascular perforation model of SAH.

\section{Acknowledgements \\ The authors would like to thank Gayle Kenney for proof reading the manuscript. \\ Author details \\ ${ }^{1}$ Laboratory of Experimental Neurosurgery, Institute for Surgical Research, Munich, Germany. '2Department of Neurosurgery, Munich, Germany. ${ }^{3}$ Institute for Stroke and Dementia Research, University of Munich Medical Center - Grosshadern, Ludwig-Maximilians-University, Munich, Germany.}

\section{Authors' contributions}

$\mathrm{KH}$ carried out the SAH experiments, performed data analysis, and drafted the manuscript. RT participated in the design and coordination of the study and supervised the experiments. KS participated in the design of the study and edited the manuscript. ET supported $\mathrm{KH}$ in performing the experiments and analyzing the data. NP initiated, designed, and coordinated the study and finalized the manuscript. All authors read and approved the final manuscript.

\section{Competing interests}

The authors declare that they have no competing interests.

Received: 15 December 2011 Accepted: 13 March 2012 Published: 13 March 2012

\section{References}

1. Bederson JB, Germano IM, Guarino L: Cortical blood flow and cerebral perfusion pressure in a new noncraniotomy model of subarachnoid hemorrhage in the rat. Stroke 1995, 26:1086-1091.

2. Prunell GF, Mathiesen T, Diemer NH, Svendgaard NA: Experimental subarachnoid hemorrhage: subarachnoid blood volume, mortality rate, neuronal death, cerebral blood flow, and perfusion pressure in three different rat models. Neurosurgery 2003, 52:165-175.

3. Schwartz AY, Masago A, Sehba FA, Bederson JB: Experimental models of subarachnoid hemorrhage in the rat: a refinement of the endovascular filament model. J Neurosci Methods 2000, 96:161-167.

4. Zausinger S, Thal SC, Kreimeier U, Messmer K, Schmid-Elsaesser R: Hypertonic fluid resuscitation from subarachnoid hemorrhage in rats. Neurosurgery 2004, 55:679-686. 
5. Zausinger S, Baethmann A, Schmid-Elsaesser R: Anesthetic methods in rats determine outcome after experimental focal cerebral ischemia: mechanical ventilation is required to obtain controlled experimental conditions. Brain Res Brain Res Protoc 2002, 9:112-121.

6. Bederson JB, Levy AL, Ding WH, Kahn R, DiPerna CA, Jenkins AL III, Vallabhajosyula P: : Acute vasoconstriction after subarachnoid hemorrhage. Neurosurgery 1998, 42:352-360.

7. Jackowski A, Crockard A, Burnstock G, Russell RR, Kristek F: The time course of intracranial pathophysiological changes following experimental subarachnoid haemorrhage in the rat. J Cereb Blood Flow Metab 1990, 10:835-849.

8. Piepgras A, Thome C, Schmiedek P: Characterization of an anterior circulation rat subarachnoid hemorrhage model. Stroke 1995, 26:2347-2352.

9. Prunell GF, Mathiesen T, Svendgaard NA: Experimental subarachnoid hemorrhage: cerebral blood flow and brain metabolism during the acute phase in three different models in the rat. Neurosurgery 2004, 54:426-436.

10. Statler KD, Alexander $H$, Vagni V, Dixon CE, Clark RS, Jenkins $L$, Kochanek PM: Comparison of seven anesthetic agents on outcome after experimental traumatic brain injury in adult, male rats. J Neurotrauma 2006, 23:97-108.

11. Stover JF, Sakowitz OW, Kroppenstedt SN, Thomale UW, Kempski OS, Flugge $G$, Unterberg AW: Differential effects of prolonged isoflurane anesthesia on plasma, extracellular, and CSF glutamate, neuronal activity, 125I-Mk801 NMDA receptor binding, and brain edema in traumatic brain-injured rats. Acta Neurochir (Wien) 2004, 146:819-830.

12. Scholler K, Trinkl A, Klopotowski M, Thal SC, Plesnila N, Trabold R, Hamann GF, Schmid-Elsaesser R, Zausinger S: Characterization of microvascular basal lamina damage and blood-brain barrier dysfunction following subarachnoid hemorrhage in rats. Brain Res 2007, 1142:237-246.

13. Torok E, Klopotowski M, Trabold R, Thal SC, Plesnila N, Scholler K: Mild hypothermia (33 degrees $\mathrm{C}$ ) reduces intracranial hypertension and improves functional outcome after subarachnoid hemorrhage in rats. Neurosurgery 2009, 65:352-359.

14. Barry KJ, Gogjian MA, Stein BM: Small animal model for investigation of subarachnoid hemorrhage and cerebral vasospasm. Stroke 1979, 10:538-541.

15. Doczi T, Joo F, Adam G, Bozoky B, Szerdahelyi P: Blood-brain barrier damage during the acute stage of subarachnoid hemorrhage, as exemplified by a new animal model. Neurosurgery 1986, 18:733-739.

16. Germano A, d'Avella D, Imperatore C, Caruso G, Tomasello F: Time-course of blood-brain barrier permeability changes after experimental subarachnoid haemorrhage. Acta Neurochir (Wien) 2000, 142:575-580.

17. Miyagi $Y$, Carpenter RC, Meguro T, Parent AD, Zhang JH: Upregulation of rho $A$ and rho kinase messenger RNAs in the basilar artery of a rat model of subarachnoid hemorrhage. J Neurosurg 2000, 93:471-476.

18. Scholler K, Feiler S, Anetsberger S, Kim SW, Plesnila N: Contribution of Bradykinin Receptors to the Development of Secondary Brain Damage After Experimental Subarachnoid Hemorrhage. Neurosurgery 2011.

19. Feiler S, Friedrich B, Scholler K, Thal SC, Plesnila N: Standardized induction of subarachnoid hemorrhage in mice by intracranial pressure monitoring. J Neurosci Methods 2010, 190:164-170.

20. Field KJ, White WJ, Lang CM: Anaesthetic effects of chloral hydrate, pentobarbitone and urethane in adult male rats. Lab Anim 1993, 27:258-269.

21. Wixson SK, White WJ, Hughes HC Jr, Lang CM, Marshall WK: The effects of pentobarbital, fentanyl-droperidol, ketamine-xylazine and ketaminediazepam on arterial blood $\mathrm{pH}$, blood gases, mean arterial blood pressure and heart rate in adult male rats. Lab Anim Sci 1987, 37:736-742.

22. Cervin A, Lindberg S: Changes in mucociliary activity may be used to investigate the airway-irritating potency of volatile anaesthetics. $\mathrm{Br} J$ Anaesth 1998, 80:475-480.

23. Doi M, Ikeda K: Airway irritation produced by volatile anaesthetics during brief inhalation: comparison of halothane, enflurane, isoflurane and sevoflurane. Can J Anaesth 1993, 40:122-126.

24. Madden JA: The effect of carbon dioxide on cerebral arteries. Pharmacol Ther 1993, 59:229-250.

25. Browning JL, Heizer ML, Widmayer MA, Baskin DS: Effects of halothane, alpha-chloralose, and pCO2 on injury volume and CSF beta-endorphin levels in focal cerebral ischemia. Mol Chem Neuropathol 1997, 31:29-42.
26. Katsura K, Kristian T, Smith ML, Siesjo BK: Acidosis induced by hypercapnia exaggerates ischemic brain damage. J Cereb Blood Flow Metab 1994, 14:243-250.

27. Fontes RB, Aguiar PH, Zanetti MV, Andrade F, Mandel M, Teixeira MJ: Acute neurogenic pulmonary edema: case reports and literature review. $J$ Neurosurg Anesthesiol 2003, 15:144-150.

28. Vespa PM, Bleck TP: Neurogenic pulmonary edema and other mechanisms of impaired oxygenation after aneurysmal subarachnoid hemorrhage. Neurocrit Care 2004, 1:157-170

29. Claassen J, Vu A, Kreiter KT, Kowalski RG, Du EY, Ostapkovich N, Fitzsimmons BF, Connolly ES, Mayer SA: Effect of acute physiologic derangements on outcome after subarachnoid hemorrhage. Crit Care Med 2004, 32:832-838.

30. Kahn JM, Caldwell EC, Deem S, Newell DW, Heckbert SR, Rubenfeld GD: Acute lung injury in patients with subarachnoid hemorrhage: incidence, risk factors, and outcome. Crit Care Med 2006, 34:196-202.

31. Wartenberg KE, Schmidt JM, Claassen J, Temes RE, Frontera JA, Ostapkovich N, Parra A, Connolly ES, Mayer SA: Impact of medical complications on outcome after subarachnoid hemorrhage. Crit Care Med 2006, 34:617-623.

32. Imai A, Steffey EP, Farver TB, Ilkiw JE: Assessment of isoflurane-induced anesthesia in ferrets and rats. Am J Vet Res 1999, 60:1577-1583.

33. Head GA, McCarty R: Vagal and sympathetic components of the heart rate range and gain of the baroreceptor-heart rate reflex in conscious rats. J Auton Nerv Syst 1987, 21:203-213.

34. Marshman LA: Cushing's'variant' response (acute hypotension) after subarachnoid hemorrhage. Association with moderate intracranial tensions and subacute cardiovascular collapse. Stroke 1997, 28:1445-1450.

35. Dickinson CJ: Reappraisal of the Cushing reflex: the most powerful neural blood pressure stabilizing system. Clin Sci (Lond) 1990, 79:543-550.

36. Cushing $\mathrm{H}$ : Concerning a definite regulatory mechanism of the vasomotor centre which controls blood pressure during cerebral compression. Bull Johns Hopkins Hosp 1901, 12:290-292.

37. Matta BF, Heath KJ, Tipping K, Summors AC: Direct cerebral vasodilatory effects of sevoflurane and isoflurane. Anesthesiology 1999, 91:677-680.

38. Baumane L, Dzintare M, Zvejniece L, Meirena D, Lauberte L, Sile V, Kalvinsh I, Sjakste N: Increased synthesis of nitric oxide in rat brain cortex due to halogenated volatile anesthetics confirmed by EPR spectroscopy. Acta Anaesthesiol Scand 2002, 46:378-383.

39. Schettini A, Furniss WW: Brain water and electrolyte distribution during the inhalation of halothane. Br J Anaesth 1979, 51:1117-1124.

40. Flynn NM, Buljubasic N, Bosnjak ZJ, Kampine JP: Isoflurane produces endothelium-independent relaxation in canine middle cerebral arteries. Anesthesiology 1992, 76:461-467.

41. Iida $\mathrm{H}$, Ohata $\mathrm{H}$, lida $\mathrm{M}$, Watanabe $\mathrm{Y}$, Dohi S: Isoflurane and sevoflurane induce vasodilation of cerebral vessels via ATP-sensitive $\mathrm{K}+$ channel activation. Anesthesiology 1998, 89:954-960.

42. McPherson RW, Kirsch JR, Tobin JR, Ghaly RF, Traystman RJ: Cerebral blood flow in primates is increased by isoflurane over time and is decreased by nitric oxide synthase inhibition. Anesthesiology 1994, 80:1320-1327.

43. Smith AL, Marque JJ: Anesthetics and cerebral edema. Anesthesiology $1976,45: 64-72$

44. Murr R, Berger S, Schurer L, Kempski O, Staub F, Baethmann A: Relationship of cerebral blood flow disturbances with brain oedema formation. Acta Neurochir Suppl (Wien) 1993, 59:11-17.

45. Theodorsson A, Holm L, Theodorsson E: Modern anesthesia and peroperative monitoring methods reduce per- and postoperative mortality during transient occlusion of the middle cerebral artery in rats. Brain Res Brain Res Protoc 2005, 14:181-190.

46. Henninger N, Heimann A, Kempski O: Electrophysiology and neuronal integrity following systemic arterial hypotension in a rat model of unilateral carotid artery occlusion. Brain Res 2007, 1163:119-129.

47. Fujii Y, Takeuchi S, Sasaki O, Minakawa T, Koike T, Tanaka R: Ultra-early rebleeding in spontaneous subarachnoid hemorrhage. J Neurosurg 1996, 84:35-42.

48. Broderick JP, Brott TG, Duldner JE, Tomsick T, Leach A: Initial and recurrent bleeding are the major causes of death following subarachnoid hemorrhage. Stroke 1994, 25:1342-1347.

49. Silverman J, Muir WW III: A review of laboratory animal anesthesia with chloral hydrate and chloralose. Lab Anim Sci 1993, 43:210-216. 
50. Grote $E$, Hassler W: The critical first minutes after subarachnoid hemorrhage. Neurosurgery 1988, 22:654-661.

doi:10.1186/2040-7378-4-5

Cite this article as: Hockel et al:: Impact of anesthesia on

pathophysiology and mortality following subarachnoid hemorrhage

in rats. Experimental \& Translational Stroke Medicine 2012 4:5.

Submit your next manuscript to BioMed Central and take full advantage of:

- Convenient online submission

- Thorough peer review

- No space constraints or color figure charges

- Immediate publication on acceptance

- Inclusion in PubMed, CAS, Scopus and Google Scholar

- Research which is freely available for redistribution

Submit your manuscript at 